\title{
Modelling the Dynamics of Vernalization: The Role of Conceptualization in Model Formulation
}

\author{
$\underline{\text { R. S. Anderssen }}^{\text {a }}$, E. J. Finnegan, C. A. Helliwell and M. Robertson ${ }^{\text {b }}$ \\ ${ }^{\text {a } C S I R O ~ D i g i t a l ~ P r o d u c t i v i t y ~ \& ~ S e r v i c e s, ~ C a n b e r r a, ~ A C T ~} 2601$ \\ ${ }^{\mathrm{b}}$ CSIRO Agriculture Business Unit, Canberra, ACT 2601 \\ Email: Bob.Anderssen@csiro.au
}

\begin{abstract}
For a plant to flower, its development must switch from the vegetative to the flowering state in order to make the seeds for the next generation. For cereals and the model plant Arabidopsis, the switching occurs in response to a cold event, signalling to the plant that it is winter with spring and summer soon to follow. Much is understood about the nature of this switching process genetically in terms of how the expression of the FLOWERING LOCUS C $(F L C)$ gene, orchestrating the vegetative development, is repressed. Only recently has a mechanism been proposed that explains how the cold initiates the repression process that switches OFF the expression of Arabidopsis FLC. This paper will discuss how the mathematics of rubber elasticity has been the conceptualization step in the formulation of a model for this proposed mechanism for the initiation, in response to a cold event, of the genetics steps that initiate the repression. In part, the goal is to use the modelling of the switching of a plant from its vegetative to its flowering phase as an illustration of the pivotal role played by the conceptualization step in model formulation.
\end{abstract}

The successful implementation of the conceptualization process in modelling and simulating real-world processes hinges on two separate matters:

The need is required not only for

(i) direct involvement, experience and familiarity with and understanding of different successful real-world (industrial) modelling endeavours,

but also for

(ii) a complete and comprehensive understanding of the workings of the particular real-world system being studied along with the motivation for and the essence of the questions to be answered.

The importance of (i) is the need for modelers to gain experience by being directly involved with the solving of real-world problems, such as industrial mathematics study group meetings and related endeavours. That of (ii) relates to the need to have a close and productive collaboration with the originators of the real-world problem that results in a win-win relationship with them.

For example, the modeler must be certain that in the conceptualization step, the outcome must not only capture the essence of the actions responsible for the observed phenomenon, it must not be inconsistent with other features, particularly those related to actions resulting from the observed phenomenon. It is also important, for a model to be truly useful, that it not only captures the observed phenomenon or event but also makes predictions of events not yet observed, which could subsequently be used to test the validity of the model. It is only with such validation that the accuracy/success of the conceptualization step be truthfully measured.

Keywords: Vernalization, conceptualization, polymer elasticity 


\section{INTRODUCTION}

When formalizing models of biological processes (such as the positioning of the trichome hairs on the leaves of plants $[12,25])$, the first step is the conceptualization [14] of the essence of the situation that becomes the aspect that must be characterized mathematically. It is the conceptualization step that performs the linking of some real-world process to an appropriate mathematical characterization which allows the questions under examination to be answered [3]. In many ways, it is the most difficult step to perform when modelling realworld processes. In part, this is because conceptualization requires both a thorough understanding of the sequential steps in the dynamics of the process under consideration, and a comprehensive involvement with situations where the conceptualization, model formulation and implementation have already been successfully performed. The required conceptualization then comes from identifying a commonality between a successful situation and the one under consideration.

A simple historical illustration comes from the observation of Fibonacci [11, 13] and others [27] that the numbers $2,3,5,8,13,21,34,55, \cdots$, which arise in biology, such as in the growth dynamics of rabbit populations, the organization of the leaves on plants and the number of petals on flowers, could be formalized algebraically as being generated by the iteration

$F_{n}=F_{n-1}+F_{n-2}, \quad F_{1}=F_{2}=1, \quad n \geq 3$.

The comprensive understanding that Fibonacci had with counting and algebraic relationships was his direct involvement with proposing and supporting the use of the Hindu-Arabic numeration system for counting and calculation, and the algebra involved with solving simple polynomial equations [11]. As detailed in Debnath [11], with respect to the population growth of rabbits, Fibonacci stated the problem to be solved as

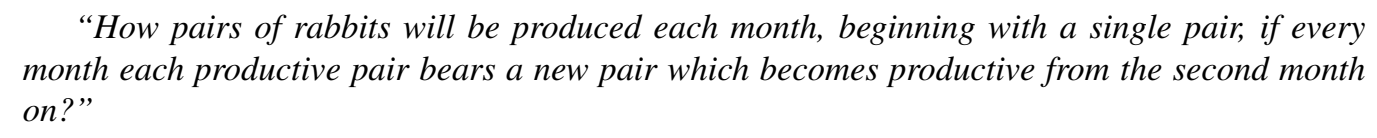

"How pairs of rabbits will be produced each month, beginning with a single pair, if every month each productive pair bears a new pair which becomes productive from the second month on?"

Consequently, starting with $1+1=2$ and working sequentially through the steps that successively generate the numbers $3,5,8,13,21,34,55, \cdots$, Fibonacci would have quickly conceptualized that he was performing the iteration 1.

"Identifying a commonality between a successful situation and the one under consideration" represents a strategy that has been proposed for enriching, humanizing and invigorating the teach of mathematics when the context has been informed by related historical examples from another field $[8,10,20]$.

The goal of this paper is to give an illustration of this conceptualization process in modelling different aspects of the vernalization process. In particular, the aim is to stress and illustrate how, for new model formulation activities, the understanding that underlies the conceptualization step comes from a familiarity with and understanding of different successful modelling endeavours, as well as a comprehensive knowledge of mathematics.

The paper has been organized in the following manner. Relevant biological background about the chromatin structure of DNA is given in Section 2.2 The role of vernalization in switching a plant from a vegetative to a flowering state is described in Section 3. Modelling regulated promoter switching experiments, used to understand the genetics of the switching from the vegetative state to flowering, is discussed in Section 3.1, while the modelling of the actual way in which the DNA responds to a cold event is considered in Section 4.

\section{BACKGROUND}

The biological dynamics of vernalization relates to how the DNA folds and compacts within the nucleus of a cell, referred to as its chromatin structure, and the changes in gene expression that follows [24]. Plants retain a memory of this response which is associated with epigenetic changes to the chromatin. The actual growth of the organs that form a plant occurs at the various meristems. These three concepts are expained in greater detail below.

\subsection{The promoter and gene expression}

The role of a gene promoter is the activation of the expression of its associated gene on receiving an appropriate signal from else where in the cell in which it is located. The promoter is the region of DNA that initiates transcription of its associated gene which corresponds to the first step in gene expression. Promoters are located near the transcription start sites of genes, on the same strand and upstream on the DNA. 


\subsection{The chromatin structure of DNA}

The length and diameter of a human DNA molecule are $\sim 2.5 \mathrm{~m}$ and $\sim 2 \mathrm{~nm}$, whereas the diameter of the nucleus within a cell, containing the DNA, is $\sim 10 \mathrm{~nm}$. In order to fit into the nucleus, the DNA molecule folds and compacts to form a specific structure, called the chromatin, the organization of which is directly related to the functioning of that cell. The nucleosome is the fundamental subunit of the chromatin with each nucleosome composed of a little less than two turns of DNA wrapped around a set of eight proteins called histones. The histone proteins that make up a nucleosome can be chemically modified. These modifications, such as trimethylation of lysine 27 on histone $\mathrm{H} 3(\mathrm{H} 3 \mathrm{~K} 27 \mathrm{me} 3)$ can affect chromatin structure and gene activity $[19,30]$. The folding and compacting in cell is achieved with the chromatin forming a dual structure consisting of either euchromatin, the region of the DNA that is open to support the functioning of the cell, which changes from cell to cell, and heterochromatin, the tightly compacted DNA not required to support the functioning.

\subsection{Epigenetics}

The genetics of an organism relates to how the DNA genes are expressed in the development and function of that organism, whereas the term epigenetics refers to other factors that control how and when each gene is expressed by altering the accessibility of a gene to the transcription machinery. The epigenetic machinery that controls gene expression is extremely complex and there are aspects that are not yet fully understood. Epigenetic responses are caused by developmental, external and/or environmental factors that switch genes $\mathrm{ON}$ and OFF and affect how cells read genes instead of being caused by changes in the DNA sequence. Further background information can be found in $[6,19,5,30]$.

\subsection{The shoot apical meristem}

The meristem is the tissue in most plants containing undifferentiated cells, the meristematic cells, which give rise to various organs of the plant and keep the plant growing. The shoot apical meristem (SAM) gives rise to organs such as the leaves and flowers.

\section{Vernalization}

As explained by Heo and Sung [18] "Vernalization is an environmentally-induced epigenetic switch in which winter cold triggers epigenetic silencing of floral repressors and thus provides competence to flower in spring."

Plants are immobile organisms and as such utilize changes in environmental conditions to optimise growth, respond to adverse conditions and commence reproductive development. A key environmental cue used to time flowering is vernalization (extended cold winter temperatures). In order to initially support their vegetative growth, vernalization-responsive Arabidopsis plants have a high level of expression of the FLOWERING LOCUS C $(F L C)$ gene, which prevents flowering from occurring.

The cold event, which triggers the vernalization response, results in the repression of the $F L C$ gene. Much is understood about the associated genetics and the consequential initiation of flowering $[2,17,21]$. This understanding has been obtained in various ways including using regulated promoter switching experiments [4]. In that situation, for the modelling of the data coming from such a switching experiment, the conceptualization step was the realization that the associated ON and OFF promoter switching corresponded causally to the use of step strain experiments to recover the relaxation moduls of linear viscoelastic materials [4].

For the modelling of the response of a plant to vernalization, the key point requiring understanding is how the physical process of a temperature change initiates the consequential genetic activity. A reductio ad absurdum argument leads to the conclusion that, in response to a cold event, if nothing physical happens, then the associated genetic events initiate spontaneously. Consequently, something physical must happen to the DNA before the genetic activity can be initiated. The fact that there are no known mutants that are able to block the repression of $F L C$ in response to a prolonged cold event represents additional support for the view that it is a physical event that activates the genetic repression of $F L C$.

Since the topology of the DNA, in terms of its chromatin structure, is its only physical characteristic, this leads to the realization that it is the topology of the DNA that changes in response to a cold event. 


\subsection{Switching from the vegetative to the flowering state}

In many plants, the transition of the shoot apical meristem from a vegetative to a flowering state occurs in response to environmental cues. Day length is one such cue, with some plants requiring short, others long, days to initiate flowering. The site for the perception of day length is the leaf, where a signal is generated and subsequently translocated to the shoot apex, on which a new pattern of cell division forms the flowering meristem that subsequently produces the flowers [7].

Vernalization, resulting from the exposure to low temperatures is another cue. It can also induce flowering in many temperate monocots and dicots including the model plant Arabidopsis. Experiments involving cold treatment to localized parts of plants have shown that the shoot apical meristem is itself a site for the perception of low-temperature treatments.

In the study of the genetics of vernalization $[17,21]$, regulated promoter switching experiments have been performed to understand the dynamics of the genetic control of vernalization in plants; in particular, regulated promoter switching experiments are used to examine the relationship between the transcription activity of Arabidopsis $F L C$ and the associated activity of $\mathrm{H} 3 \mathrm{~K} 27 \mathrm{me} 3$ at this vernalization-responsive gene in plants.

The experimental protocol was designed so that the transcription of $F L C$ can be abruptly switched ON and OFF using a promoter system (pOp6) regulated by the application of dexamethasone [9]. In this way, the $F L C$-H3K27me3 chromatin modification activity can be assessed in terms of how the level of H3K27me3 changes in response to the switching $\mathrm{ON}$ or OFF of FLC transcription.

The abrupt switching ON and OFF of a process, in order to understand the underlying phenomenology, is also used in the study of the stress-strain response of linear viscoelastic materials. The underlying causal (Boltzmann, Volterra) model takes the form

$\sigma(t)=\int_{0}^{t} G(t-\tau) \frac{d \gamma(\tau)}{d \tau}$

where $\sigma(t), G(t)$ and $\gamma$ denote, respectively, the measured stress response, the relaxation modulus and the applied strain. For the direct experimental recovery of an estimate of $G(t)$, the strain $\gamma$ is abruptly switched on, which corresponds to taking $\gamma(t)=H(t)$, the Heaviside unit step function. Taking account of the fact that the time derivative of $H(t)$ is the Dirac delta function $\delta(t)$, equation (2) yields on

$$
\sigma(t)=G(t)
$$

It is this direct experimental recovery of an estimate of the relaxation modulus that is the basis for the importance and popularity of the step strain experiment.

Consequently, as proposed and exploited in [4], with respect to the regulated promoter experiment, the conceptualization is that the measured response to the switching ON or OFF represents a characterization, like the relaxation modulus for a viscoelastic material, of the relationship between the $F L C$-H $3 \mathrm{~K} 27 \mathrm{me} 3$ chromatin modification activity.?????

\section{THE INITIATION OF VERNALIZATION BY THE COLD}

Having identified that the only physical aspect of DNA that can change is its topology, the next step in the conceptualization process is the identification as to how a cold event causes this the topology to change.

The topology of DNA is quite flexible in terms of its chromatin structure [28], the primary functions of which are (1) to package DNA into a smaller volume to fit in the cell, (2) to reinforce the DNA macromolecule to allow mitosis, (3) to prevent DNA damage, and (4) to control gene expression and DNA replication. The flexibility is achieved through DNA having a polymer structure with the repeat unit, corresponding to the Kuhn length in synthetic polymers, being the nucleosomes [22, 28].

Since a cold event corresponds to a temperature change, the conceptualization step reduces to identifying how a temperature event changes the topology of a polymer. In rheology, the elasticity $E_{\text {ent }}$ of a polymer is known to depend on the temperature $T$ with the underlying mechanism being the entropic state of the Kuhn lengths $b$ and the contour length $L_{c}$ under consideration (e.g. equation (2) in [29] and equation (9) in [23])

$E_{\text {ent }}=K \frac{T}{L_{c} b}$ 

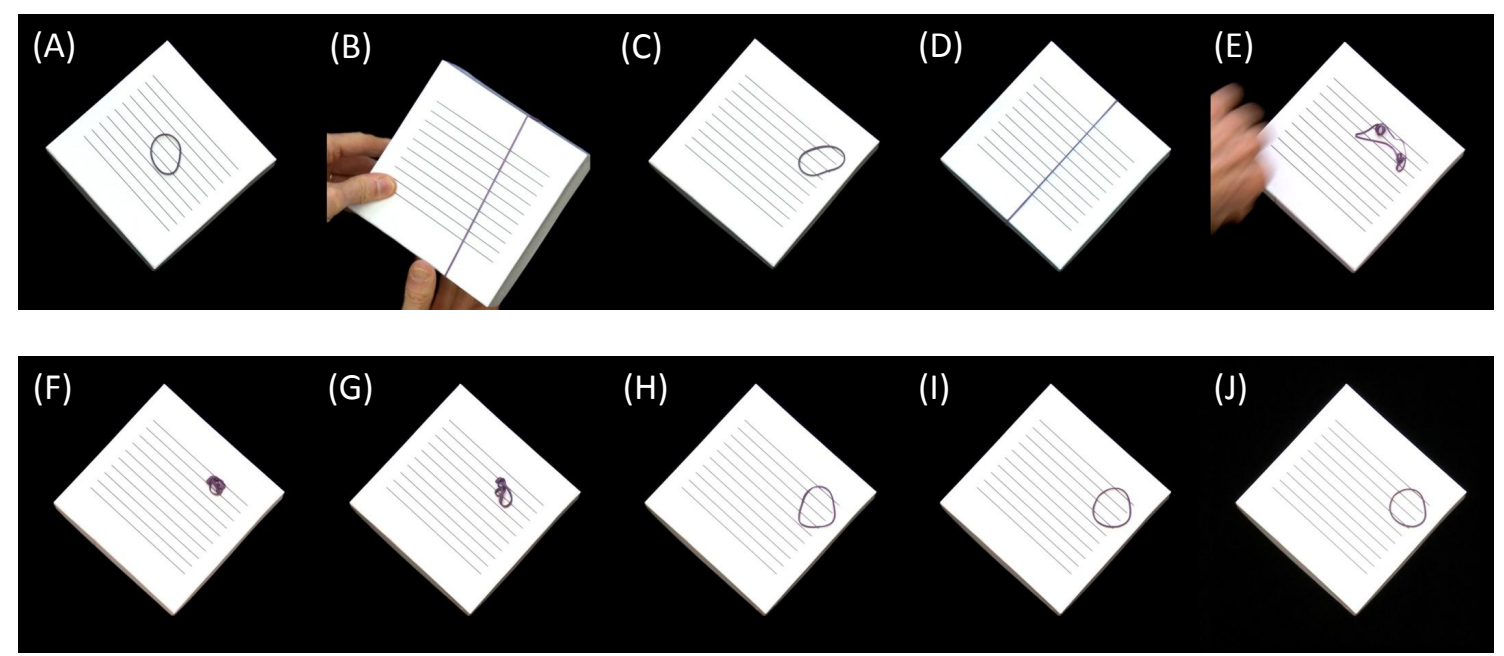

Figure 1: A rubber band relaxes when placed at freezing temperatures due to changes in the entropic state of its polymer structure. The panels shown are still shots from a video taken to record the response of a rubber band to temperature change: (A) The rubber band before being put around the box; (B) The rubber band is put onto the box at room temperature; (C) The rubber band immediately after being taken off the box at room temperature; (D) The band is put back onto the box and placed at -20C; (E) (J) Time course showing the band contracting after being removed from the box: image taken at 0 seconds (E); 5 seconds (F); 3 minutes 54 secs $(\mathrm{G}) ; 4$ minutes 24 seconds $(\mathrm{H}) ; 5$ minutes 30 seconds $(\mathrm{I}) ; 10$ minutes $(\mathrm{J})$.

Consequently, as explained in [16], for DNA, the conceptualization step is to assume that a similar phenomenology holds with the initial response to a temperature event being an entropically-induced reorganization in the folding or looping of both the euchromatin and the hetrochromatin. In fact, because polymer entropy responds to temperature, the organization of its chromatin in the cold will be more relaxed, potentially disrupting higher order chromatin folding and looping [16].

The importance of folding and looping of chromatin in gene expression dynamics is fairly well understood $[15,31]$. At higher temperatures, it will tend to be more strongly randomly folded than at lower temperatures. The prototypical example is the effect of temperature on an elastic band $[1,26]$, which can be demonstrated by the following experiment. An elastic rubber band is placed around a box and the whole package is the put into a freezer for at least an hour. On being removed from the freezer, the rubber band is removed from the box. The elastic band contracts back to its original configuration in response to its thermodynamic state moving to one which is higher. This is illustrated in Figure 1.

Interestingly, the role of increasing entropy also holds for the heating of a metal rod. In that situation, as the rod is heated, it expands leading to a greater disorganization within its crystalline structure.

\subsection{The resulting modelling opportunities}

The importance of equation (3) is that it represents a starting point for the formulation of a model with respect to the question under examination. For example, if the question under consideration relates to how the looping and folding of the DNA changes locally as the temperature changes, one can assume that equation (3) generalizes locally to take the form

$E_{\text {ent }}(T)=K \frac{T}{L_{c}(T) b(T)}$,

where it is now assumed that the contour length and the Kuhn length change with temperature. Justification for this step can be based on the known behaviour of DNA having different topological configurations such as is found in the euchromatin and heterochromatin.

Acknowledgements. The author thanks the reviewers for their comments that assisted with improving the clarity of the deliberations within the paper. 


\section{REFERENCES}

[1] G. Allen, M. J. Kirkham, J. Padget, and C. Price. Thermodynamics of rubber elasticity at constant volume. Trans. Faraday Soc., 67:1278-\&, 1971.

[2] R. Amasino. Vernalization, competence, and the epigenetic memory of winter. The Plant Cell, 16:25532559, 2004.

[3] R. S. Anderssen. The pragmatics of solving industrial (real-world) inverse problems with exemplification based on the molecular weight distribution problem. Inverse Problems, 15:R1-R40, 1999.

[4] R. S. Anderssen and C. A. Helliwell. Information recovery in molecular biology: causal modelling of regulated promoter switching experiments. J. Math. Biology, 67:105-122, 2013.

[5] S. R. Bhaumik, E. Smith, and A. Shilatifard. Covalent modifications of histones during development and disease pathogenesis. Nature structural \& molecular biology, 14:1008-1016, 2007.

[6] A. Bird. Perceptions of epigenetics. Nature, 447:396-398, 2007.

[7] J. E. Burn, D. J. Bagnall, J. D. Metzger, E. S. Dennis, and W. J. Peacock. DNA methylation, vernalization, and the initiation of flowering. Proceedings of the National Academy of Sciences, 90:287-291, 1993.

[8] R. Calinger. Vita mathematica: Historical research and integration with teaching, volume 40. Cambridge University Press, 1996.

[9] J. Craft, M. Samalova, C. Baroux, H. Townley, A. Martinez, I. Jepson, M. Tsiantis, and I. Moore. New pOp/LhG4 vectors for stringent glucocorticoid-dependent transgene expression in Arabidopsis. Plant J., 41:899-918, 2005.

[10] M. A. B. Deakin. Using history to teach mathematics. Zentralblatt für Didaktik der Mathematik, 33:137$138,2001$.

[11] L. Debnath. A short history of the fibonacci and golden numbers with their applications. International Journal of Mathematical Education in Science and Technology, 42:337-367, 2011.

[12] M. P. Edwards, S. Pereverzyev Jr., and R. S. Anderssen. Modelling pattern formation in plants. In MODSIM2011, 19th International Congress of Modelling and Simulation, pages 378-384. Modelling and Simulation Society of Australian and New Zealand, 2011.

[13] H. W. Gould. A history of the fibonacci q-matrix and a higher-dimensional problem. Fibonacci Quart, 19:250-257, 1981.

[14] B. Greer. Modelling reality in mathematics classrooms: The case of word problems. Learning and instruction, 7:293-307, 1997.

[15] D. W. Heermann. Physical nuclear organization: loops and entropy. Current opinion in cell biology, 23:332-337, 2011.

[16] C. A. Helliwell, R. S. Anderssen, M. Robertson, and E. J. Finnegan. How is FLC repression initiated by cold? Trends in Plant Science, 20:76-82, 2015.

[17] J. B. Heo and S. Sung. Encoding memory of winter by noncoding RNAs. Epigenetics, 6:544-547, 2011.

[18] J. B. Heo and S. Sung. Vernalization-mediated epigenetic silencing by a long intronic noncoding RNA. Science, 331:76-79, 2011.

[19] T. Jenuwein and C. D. Allis. Translating the histone code. Science, 293:1074-1080, 2001.

[20] V. J. Katz. Using history to teach mathematics: An international perspective, volume 51. Cambridge University Press, 2000.

[21] D.-H. Kim, M. R. Doyle, S. Sung, and R. M. Amasino. Vernalization: Winter and the Timing of Flowering in Plants. Ann. Rev. Cell Dev. Biol., 25:277-299, 2009.

[22] R. D. Kornberg. Structure of chromatin. Annual review of biochemistry, 46:931-954, 1977. 
[23] T. C. B. McLeish. Tube theory of entangled polymer dynamics. Advances in Physics, 51:1379-1527, 2002.

[24] C. Mozzetta, E. Boyarchuk, J. Pontis, and S. Ait-Si-Ali. Sound of silence: the properties and functions of repressive lys methyltransferases. Nature Reviews Molecular Cell Biology, 16:499-513, 2015.

[25] C. M. OKeefe, S. Pereverzyev Jr, and R. S. Anderssen. The algebra of hexagonal numbers. The Mathematical Scientist, 36:1-9, 2011.

[26] D. Roundy and M. Rogers. Exploring the thermodynamics of a rubber band. American Journal of Physics, 81:20-23, 2013.

[27] P. Singh. The so-called fibonacci numbers in ancient and medieval india. Historia Mathematica, 12:229244, 1985.

[28] S. B. Smith, Y. J. Cui, and C. Bustamante. Overstretching B-DNA: The elastic response of individual double-stranded and single-stranded DNA molecules. Science, 271:795-799, 1996.

[29] S. B. Smith, L. Finzi, and C. Bustmante. Direct mechanical measurements of the elasticity of single DNA-molecules by using magnetic beads. Science, 258:1122-1126, 1992.

[30] B. D. Strahl and C. D. Allis. The language of covalent histone modifications. Nature, 403:41-45, 2000.

[31] M. Tark-Dame, R. van Driel, and D. W. Heermann. Chromatin folding - from biology to polymer models and back. J. Cell Science, 124:839-845, 2011. 